\title{
La Evaluación Docente en la Universidad: Visiones de los Alumnos
}

\author{
The Teaching Evaluation in the University: Views of the \\ Students
}

\author{
Tiburcio Moreno Olivos * \\ Universidad Autónoma Metropolitana, México
}

\begin{abstract}
Este trabajo pretende contribuir a una mejor comprensión del proceso de evaluación del desempeño docente en el contexto universitario, evidenciando la inoperancia y poca validez del cuestionario como mecanismo para el perfeccionamiento de la educación superior. El artículo tiene como propósito analizar las perspectivas de los alumnos de licenciatura (pregrado) respecto al cuestionario de opinión con el que valoran periódicamente el desempeño de sus profesores, para juzgar su pertinencia y utilidad como instrumento de evaluación. Se trata de una investigación de corte cualitativo mediante la modalidad de estudio de casos. El caso estuvo representado por las Unidades Xochimilco y Cuajimalpa de la Universidad Autónoma Metropolitana (UAM). La perspectiva teórica de la evaluación docente también se sitúa en un marco de evaluación cualitativa y comprensiva. Los resultados se agrupan en tres bloques referidos al cuestionario de evaluación docente que los alumnos responden: 1) opiniones del alumnado sobre el cuestionario, 2) usos de los resultados de la evaluación docente, y 3) propuesta para evaluar el desempeño docente. Entre las conclusiones se destaca que el proceso de evaluación está asociado de forma prioritaria con el otorgamiento de estímulos económicos a los profesores y tangencialmente con la mejora de la calidad de la enseñanza. Esta situación discrepa con una visión muy extendida que vincula los resultados de la evaluación docente con el mejoramiento de la educación.
\end{abstract}

Descriptores: Evaluación; Docente; Estudiante; Enseñanza superior.

The purpose of this paper is to contribute to a better understanding of the process of evaluation of teaching performance in the university context, evidencing the ineffectiveness and poor validity of the questionnaire as a mechanism for the improvement of higher education. The paper analyzes the perspectives of undergraduate students about a questionnaire with which they periodically assess the performance of their professors, to judge their relevance and usefulness as an evaluation tool. It is a qualitative research through the case study modality. The case was represented by the Xochimilco and Cuajimalpa Units of the Autonomous Metropolitan University (UAM). The theoretical perspective of teacher evaluation is also placed in a framework of qualitative and comprehensive evaluation. The results are grouped into three blocks referring to the teacher evaluation questionnaire that the students answer: 1) student opinions on the questionnaire, 2) uses of the results of the teacher evaluation, and 3) proposal to evaluate the teaching performance. Among the conclusions, it is emphasized that the evaluation process is associated in a priority way with the granting of economic stimuli to teachers and tangentially with the improvement of the quality of teaching. This situation disagrees with a very extended vision that links the results of the teacher evaluation with the improvement of education

Keywords: Evaluation; Teachers; Students; Higher education.

*Contacto: tmoreno@correo.cua.uam.mx

ISSN: 1696-4713

www.rinace.net/reice/

revistas.uam.es/reice
Recibido: $\quad 7$ de enero 2018

$1^{\text {a }}$ Evaluación: 13 de marzo 2018

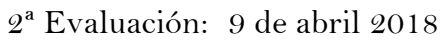

Aceptado: $\quad 6$ de junio 2018 


\section{Introducción}

La calidad de la educación superior depende, entre otros factores, de la calidad de la enseñanza y, ésta, a su vez, se ve fuertemente influida por la calidad del docente. En las tres últimas décadas las instituciones de educación superior (IES) mexicanas han emprendido una serie de reformas orientadas a lograr la calidad de la educación. Una estrategia que se ha empleado para conseguir tan importante propósito ha sido la evaluación de la docencia. "En el mundo contemporáneo la evaluación docente ha cobrado un papel muy relevante en dos sentidos: evaluación para la rendición de cuentas y evaluación para la mejora" (Cuevas y Moreno, 2016, p. 5). No obstante, la evaluación desde su origen ha estado ligada al otorgamiento de estímulos económicos, de modo que los profesores que consiguen resultados positivos en su evaluación se hacen acreedores a ciertos emolumentos, que en algunos casos representan una parte muy significativa de sus ingresos económicos (Comas y Lastra, 2010). En cambio, aquellos que obtienen resultados negativos en su evaluación se ven excluidos de tales beneficios. Se trata, entonces, de una evaluación de pago al mérito (merity pay), que distribuye premios y castigos; es una evaluación punitiva que genera relaciones de competitividad entre el profesorado, lo cual atenta contra las iniciativas que buscan promover la colegialidad y la colaboración docente (Hargreaves, 1996; Moreno, 2006).

Por otro lado, a pesar de los avances teórico-metodológicos de la evaluación como disciplina y campo de investigación, la evaluación de la docencia en México ha estado dominada por un paradigma de investigación de corte psico-estadístico en el que los resultados cuentan más que los procesos. Este tipo de evaluaciones mide lo que es más fácil de medir y no necesariamente lo que es más importante para el desarrollo y progreso de la enseñanza. Un problema central estriba en que determinadas formas de evaluación sólo pueden valorar ciertos tipos de contenidos de aprendizaje, con lo cual es posible que se enfoquen sobre lo irrelevante y orienten la práctica docente hacia cuestiones triviales.

Al parecer, la razón primordial por la que prácticamente la totalidad de las IES emplea un "cuestionario de opinión" como estrategia principal para valorar el desempeño del personal docente, es el tamaño y la complejidad del sistema de educación superior mexicano. El uso de un cuestionario estandarizado resulta más económico (aunque no tanto como se suele pensar) que otras técnicas e instrumentos de valoración de corte cualitativo. No obstante, con el paso del tiempo se ha observado que los alumnos contestan el cuestionario de forma rutinaria y, en ocasiones, con cierta actitud de indiferencia ante la certeza de que sus respuestas no tendrán ningún efecto que genere un cambio real en la enseñanza. Este ejercicio se ha convertido en un trámite burocrático administrativo, despojando así a la evaluación de su función formativa y cancelando toda posibilidad de mejora significativa de la enseñanza y el aprendizaje de los alumnos (Rueda y Díaz Barriga, 2011). Además, el equiparar a la evaluación docente con un cuestionario de opinión es, por decir lo menos, restrictivo y distorsionador del verdadero sentido del proceso de evaluación.

Este artículo se centra en la evaluación del profesorado de educación superior y busca contribuir al debate conceptual del campo ante la ausencia de un marco teórico claramente definido y compartido por la comunidad científica que investiga este tema. Se considera que para comprender la problemática de la evaluación de los profesores es perentorio el análisis y discusión de algunos conceptos clave propios de este ámbito. Para ello, el 
documento se estructura en cuatro apartados: a) Fundamentación teórica; b) Método; c) Resultados y Discusión; y d) Conclusiones.

\section{Fundamentación teórica}

\subsection{Descripción de la problemática}

En años recientes se nota un marcado interés en todo el mundo por conseguir una enseñanza de calidad y para ello la evaluación es vista como una herramienta primordial. Pero no vale cualquier tipo de evaluación, se requiere de una "buena evaluación", condición que no siempre resulta fácil de satisfacer.

Por otra parte, es innegable la relevancia que la evaluación de los maestros ha alcanzado en las últimas décadas en México, siendo uno de los asuntos más complejos, polémicos y controvertidos de la actual reforma educativa. Aunque esta preocupación por la evaluación docente no es nueva, es en años recientes que ha tenido su punto más álgido.

Aunque en el plano discursivo se reconoce la importancia que el proceso evaluativo tiene para los participantes, es habitual que no se conceda la atención y el rigor que dicho proceso requiere para que resulte valioso. En general, las prácticas evaluadoras en el sistema educativo mexicano se suelen caracterizar por la superficialidad, la homogeneidad y un exiguo conocimiento teórico-metodológico por parte de los encargados de su diseño e implementación. Justamente la evaluación docente es un claro ejemplo del mal uso que en ocasiones se ha hecho tanto del proceso como de sus resultados, lo cual lejos de contribuir a una mejora de la educación ha producido el efecto contrario, lesionando la motivación y generando desconfianza hacia la evaluación misma. Una vez que la evaluación ha sido desacreditada y puesta en tela de juicio, es previsible que la credibilidad y validez de sus resultados no sean considerados con seriedad por parte de los evaluados (Moreno, 2010).

Con el devenir del tiempo la evaluación del profesorado ha captado la atención de diversos investigadores, quienes se han interesado en ella como objeto de estudio, señalado algunos de sus logros y problemas más acuciantes (Cordero y González, 2016; Murillo, 2007; Tejedor y Jornet, 2008). Sin embargo, particularmente en el contexto mexicano, buena parte de los trabajos referidos a la evaluación de los docentes de educación superior presentan como limitante principal la carencia de un sólido marco teórico que les sirva de sustento. Se trata, sobre todo, de estudios de corte descriptivo, que tienen un sesgo psicológico e ignoran o abordan escasamente el contexto (entendido en sentido amplio) en el que los profesores llevan a cabo sus prácticas pedagógicas. Dichos estudios comúnmente dan por sentado su objeto de estudio y no suelen explicitarlo (condición sine qua non de cualquier evaluación que se precie de serlo) o lo hacen de manera imprecisa, lo que genera ambigüedad con respecto a lo que realmente se pretende valorar del quehacer de los docentes.

\subsection{La mejora de la evaluación docente: Un tema escurridizo}

En las IES mexicanas existe una percepción generalizada de que la evaluación docente necesita ser mejorada, pero al parecer nadie está dispuesto a actuar para cambiar el sistema vigente. Pervive cierta insatisfacción con la evaluación y esto es así, entre otras razones, porque el esquema actual raramente ayuda a los maestros a mejorar su enseñanza, y no permite distinguir con claridad a los que sobresalen de aquellos que están luchando o 
esforzándose por conseguirlo. Los instrumentos de evaluación no representan los rasgos sustanciales de una "buena enseñanza". Además, falta discutir y consensar cuestiones como: ¿qué es una buena enseñanza?, ¿quién define la buena enseñanza?, ¿buena enseñanza para qué y para quién?, ¿es posible evaluar la calidad de la enseñanza sin la existencia de un marco de buenas prácticas para la educación superior en el país? Se admite que no existe ninguna definición inequívoca de la enseñanza, válida en todo tiempo y lugar. Que la visión de la enseñanza que hoy parece satisfactoria en nuestra sociedad puede no ser la definición aceptada en otro tiempo o en otra cultura (Jackson, 2002).

La bibliografía especializada ha reconocido algunas dificultades que afrontan los actuales sistemas de evaluación, en un informe reciente sobre evaluación docente un grupo de profesores expertos - The Accomplished California Teachers Network (La Red de Profesores Expertos de California)- identificó que algunos de sus distritos habían tenido una serie de problemas en los procedimientos de evaluación, tales como: 1) carencia de consistencia y de estándares claros de lo que es una buena práctica; 2) no centrar el foco en la mejora de la práctica; 3) tiempo y personal inadecuados para realizar evaluaciones efectivas; 4) poca o ninguna consideración de los resultados de los alumnos; 5) procedimiento del "cortador de galletas" ${ }^{1}$ que no considera las necesidades del profesor; y 6) evaluaciones separadas del desarrollo profesional (Darling-Hammond, 2013). Es patente que casi todos estos problemas aplican para el caso de la evaluación del profesorado de educación superior en México.

\subsection{La evaluación docente y su importancia}

La expresión "evaluación docente" es polisémica, es decir, que tiene diversos significados, pero en este artículo es concebida como el proceso de describir y juzgar los méritos y la valía de los profesores en función de sus conocimientos, destrezas, conducta y los resultados de su enseñanza. La evaluación docente cumple distintas funciones, algunas veces se emplea para controlar a los profesores, para motivarlos, para que se responsabilicen de su trabajo, o para liberarse de ellos cuando no lo realizan bien. En ocasiones, los resultados de la evaluación sirven como argumento para justificar la criba de los docentes (Nevo, 1997). Sin embargo, en la educación superior mexicana este tamiz de la evaluación generalmente no aplica para el profesorado, quien independientemente de sus resultados no verá amenazado su puesto de trabajo, en el peor de los casos será excluido de recibir un estímulo económico; seguramente ocurre algo distinto con los docentes del sector privado cuya permanencia en su puesto o la renovación de su contrato laboral penden de los resultados de la evaluación docente. Esta situación ha dado pie a prácticas de evaluación perversas y simuladas, como la cooptación de los alumnos por parte de ciertos profesores para salir bien librados de la evaluación.

Así, Nevo (1997) identifica como funciones de la evaluación del profesorado las siguientes: a) desarrollar y asegurar la competencia docente, b) otorgar certificados a los profesores, c) contratar nuevos profesores, d) mejorar la docencia, e) responsabilizarse ante alumnos, padres y comunidades, f) negociar un acuerdo de evaluación, y g) obtener reconocimiento o certificación nacional. ${ }^{1}$ La expresión "cortador de galletas" alude a un procedimiento de evaluación docente que se aplica de forma homogénea
para todos, sin considerar las condiciones y particularidades de cada docente. 
Desde hace mucho tiempo en el ámbito internacional parece existir cierto consenso en el sentido de que sin el compromiso e implicación del profesorado ningún proyecto de cambio o reforma educativa es viable (Escudero, 2002; Fullan, 1993; Murillo, 2003). La forma más directa en que los profesores y sus alumnos se pueden beneficiar de la evaluación del profesorado es el hecho de que proporciona información acerca del modo en que éstos enseñan y en el que aprenden sus alumnos. Tal evaluación puede ayudar a los profesores a mejorar su docencia según las necesidades de los alumnos, y así mejorar el aprendizaje de éstos. Así, se afirma que,

\begin{abstract}
para apoyar la enseñanza para la comprensión y el desarrollo profesional que ésta implica, será necesario que las nuevas formas de evaluación de maestros se adecuen a los propósitos y contextos de la enseñanza y a las necesidades de los estudiantes. $\mathrm{ra}$ no es suficiente enfocarse a que los maestros se ajusten a las rutinas prescritas. La evaluación debe concebirse no como un evento anual que consiste en breves visitas por parte de los supervisores y en el chequeo de un listado, sino en un recurso constante para los practicantes sobre la organización y el ambiente dentro del aula. (DarlingHammond y McLaughlin, 2003, p. 26)
\end{abstract}

Aunque la evaluación docente ha recibido numerosas críticas de diversa índole, esto no significa que debamos rechazarla; por el contrario, reconocemos el valor y la necesidad de evaluar la calidad docente y la calidad de la enseñanza, sólo que habrá que empezar por aceptar que la forma en que se ha estado haciendo durante décadas ha contribuido muy poco -si es que en algo ha contribuido- a la mejora de la enseñanza y el aprendizaje de los alumnos. Es crucial que la función de mejora de la docencia, mediante su evaluación, se lleve a cabo de manera constructiva y no amenazadora. Por lo tanto, se debería motivar a los profesores a que sean ellos los que tomen la iniciativa de buscar y utilizar la evaluación para la auto-mejora sin esperar a que sean otros agentes externos los que se la impongan.

La evaluación es un proceso decisivo por ser considerada una vía para acceder a un conocimiento efectivo acerca de la calidad de la enseñanza impartida. En este punto quizá conviene enfatizar que la calidad del docente está inextricablemente unida a la calidad de la enseñanza, "pues tanto la experiencia como la investigación han confirmado que el factor clave para conseguir una educación de calidad es contar con docentes de calidad” (Murillo, 2007, p. 15). Sin embargo, es obvio que no se trata de una relación causal toda vez que un docente competente no siempre puede garantizar una enseñanza de calidad, en tanto que un ambiente educativo favorable puede propiciar que un profesor poco hábil libre bien las tareas de enseñanza.

\title{
1.4. ¿Calidad del docente o calidad de la enseñanza?
}

Las definiciones dominantes sobre la calidad del docente suelen inclinarse hacia el ahistoricismo y los argumentos que las utilizan prefieren afirmar antes que argumentar. No suelen propugnar por una visión de la calidad del docente, sino que más bien se conforman con una descripción evidente en sí misma. La calidad del docente no se puede analizar con efectividad si se define de una forma reductora, ahistórica o esencialista. La calidad del docente ha sido un concepto controvertido que, ciertamente, no es neutral ni evidente en sí mismo.

La calidad del docente se concibe como "el conjunto de rasgos personales, habilidades, y conocimientos que un individuo aporta a la enseñanza, incluidas disposiciones para comportarse de ciertas maneras" (Darling-Hammond, 2013, p. 11). La autora citada afirma que la investigación sobre la eficacia docente, basada en las cualificaciones del profesor y 
los logros obtenidos por el alumno, ha encontrado que son importantes las siguientes cualidades:

- Fuerte conocimiento del contenido relacionado con lo que se va a enseñar.

- Conocimiento acerca de cómo enseñar a otros en esa área (pedagogía del contenido) y habilidad para implementar prácticas de enseñanza productiva.

- Comprensión de los alumnos y su desarrollo, considerando cómo apoyar a los que tienen diferencias o dificultades en su aprendizaje y cómo apoyar el aprendizaje del lenguaje y el contenido de aquellos que no son competentes en el lenguaje de la enseñanza.

- Habilidades generales para organizar y explicar ideas, así como para observar y pensar diagnósticamente.

- Experiencia adaptativa que permita a los profesores hacer juicios acerca de lo que puede funcionar en un contexto dado y en respuesta a las necesidades de los alumnos.

Mientras que la calidad de la enseñanza se "refiere a la sólida enseñanza que permite aprender a un amplio rango de alumnos. Tal enseñanza cubre las demandas de la disciplina, las metas de enseñanza, y las necesidades de los alumnos en un contexto particular" (Darling-Hammond, 2013, p. 12). La calidad de la enseñanza es en parte una función de la calidad del docente -conocimiento, habilidades, y disposiciones- pero ésta también está fuertemente mediada por el contexto de enseñanza, toda vez que contiene factores externos a lo que el profesor sabe y puede hacer. Lo anterior quiere decir que no se puede juzgar la calidad de la enseñanza ignorando el contexto en el que ésta ocurre. Por contexto se entiende mucho más que el ámbito físico de la actividad, se refiere a un contexto cultural que comprende nociones, supuestos previos, expectativas y demás componentes que influyen en la actividad de enseñanza. Por su parte Cordero, Luna y Patiño (2013), apoyándose en un trabajo anterior de García (2008), definen la práctica docente como el conjunto de situaciones áulicas que configuran el quehacer del docente y de los alumnos en función de determinados objetivos formativos.

Según Cordero, Luna y Patiño (2013), la práctica docente se manifiesta en tres momentos: lo que pasa antes de la acción didáctica (incluye las teorías asumidas, creencias y conocimientos de la enseñanza, planeación de la clase y expectativas acerca del grupo); lo que acontece durante la interacción didáctica (involucra a las teorías en uso, realización de la situación, mecanismos de interacción y concreción de marcos referenciales del docente); y el análisis de resultados o reflexión (logros de aprendizaje, transformaciones en los aprendices y en el profesor). En este tenor, cuando se hace referencia a la evaluación de la docencia es necesario dilucidar qué es aquello que se pretende evaluar: la calidad del docente, la calidad de la enseñanza, la calidad de la práctica docente, o todas estas cuestiones juntas, pues tal imprecisión puede provocar errores en el diseño metodológico de los instrumentos para la recolección de los datos, entre otros.

Además, el reconocimiento del valor que tiene el contexto en la evaluación de la calidad de la enseñanza, significa considerar como elementos esenciales el currículo y los sistemas de evaluación que sustentan el trabajo del profesor, el ajuste entre las cualidades del profesor y lo que se le pide que enseñe, y las condiciones de enseñanza. Como se sugirió anteriormente, un excelente profesor puede ser incapaz de garantizar una enseñanza de calidad en un contex to en el que se le solicita que imparta un currículo defectuoso o carente 
de materiales apropiados. Asimismo, un profesor bien preparado puede tener un desempeño deficiente cuando se le demanda que enseñe fuera de su campo formativo o disciplinar (algo bastante habitual en el sistema educativo mexicano) o bajo condiciones precarias de enseñanza (por ejemplo, sin los materiales didácticos adecuados, con escaso tiempo o con grupos-clase demasiado grandes). Por el contrario, un profesor poco habilitado puede salir airoso si cuenta con excelentes materiales, un sólido apoyo de sus colegas para planear la clase y especialistas adjuntos que trabajan con aquellos alumnos que pueden necesitar de ayuda adicional para superar ciertas carencias en su aprendizaje (Darling-Hammond, 2013).

Desde una perspectiva sociológica la calidad de la enseñanza también ha sido analizada como una construcción social, como un intento por hacer visibles y explícitos los imperativos prácticos y de gestión ideológica en cualquier momento dado. La calidad de la enseñanza es un concepto dinámico que ha tenido diferentes significados a través del tiempo, "la calidad se redefine de acuerdo con los propósitos del Estado, a medida que se desarrollan nuevos sectores de escolarización, como sucede con la escasez, se define el trabajo para que se adapte a los grupos de probables reclutas” (Lawn y Ozga, 2004, p. 77). Desde esta perspectiva, no existe ninguna otra calidad docente que las "cualidades" personales y éstas se definen de un modo casi estereotipado.

Aunque el Estado ha definido más estrechamente la calidad del docente a medida que transcurre el tiempo, se aprecian discrepancias u omisiones entre las distintas definiciones. También existen tensiones entre la visión ideológica del docente y la práctica, así como entre la visión dominante del "buen docente" y la definición del docente. Hay una visión del docente moderno, al que se le denomina como "profesional reflexivo", que describe una meticulosa formación en un modo de enseñanza de alta habilidad basado en la indagación en clase. Esta idea se relaciona con la del docente efectivo (Lawn y Ozga, 2004).

\section{Método}

Este artículo tiene como objetivo analizar las perspectivas de los alumnos de licenciatura respecto al cuestionario de opinión con el que valoran periódicamente el desempeño de sus profesores, para juzgar su pertinencia y utilidad como instrumento de evaluación. Se trató de una investigación de corte cualitativo mediante la modalidad de estudio de casos. El caso estuvo representado por las Unidades Xochimilco y Cuajimalpa de la Universidad Autónoma Metropolitana (UAM), específicamente de las Divisiones de Ciencias y Artes para el Diseño de la Universidad Autónoma Metropolitana-Xochimilco (UAM-X) y de Ciencias de la Comunicación y Diseño de la Universidad Autónoma MetropolitanaCuajimalpa (UAM-C). La selección del caso como objeto de investigación responde a la amplia tradición en la evaluación del profesorado que ambas unidades de la UAM tienen, después de varias décadas de aplicar el cuestionario a los alumnos como instrumento de evaluación docente, así como las facilidades para acceder a la comunidad académica y realizar el levantamiento de los datos empíricos. Además, este caso permite profundizar en el tema, ampliando los resultados de otros trabajos de investigación en el contexto universitario los cuales revelan que "la evaluación docente es sinónimo de los cuestionarios estudiantiles, una rutina burocrática, parte de una remuneración económica, evaluación que es rechazada por los alumnos" (Arbesú y García, 2017, p. 1). 
Para el trabajo de campo se realizaron entrevistas a profundidad a diversas autoridades de la División de Ciencias y Artes para el Diseño de la UAM-X (director de la División, jefes de departamento, coordinadores de licenciatura y rectora de Unidad). Otro instrumento que se aplicó fue un cuestionario con preguntas abiertas dirigido a alumnos de las dos Unidades de las Divisiones académicas antes referidas, con la finalidad de conocer sus visiones acerca del cuestionario con el que periódicamente evalúan a sus profesores. Se realizó un muestreo intencionado, los criterios relevantes para la selección de los participantes fueron: a) alumnos de la carrera de Ciencias de la Comunicación; y b) que cursaran trimestres IX a XI, toda vez que para la investigación interesaban las opiniones de los alumnos de cursos avanzados que tuvieran suficiente experiencia en responder el cuestionario de evaluación docente. El $60 \%$ de los que respondieron fueron varones y el $40 \%$ mujeres, cuyas edades fluctúan entre 21 y 23 años de edad. Es preciso advertir que en este trabajo únicamente se presentan los resultados del cuestionario suministrado a los alumnos de Ciencias de la Comunicación de la UAM-Cuajimalpa, es decir, contiene sólo una parte de los hallazgos de un estudio más amplio. Los datos procedentes de las entrevistas no se incluyen en este documento.

\section{Resultados}

Los resultados se agruparon en tres bloques referidos al cuestionario de evaluación docente que los alumnos responden: 1) opiniones del alumnado sobre el cuestionario, 2) usos de los resultados de la evaluación docente, y 3) propuestas para evaluar el desempeño docente.

\subsection{Opiniones del alumnado sobre el cuestionario}

Prácticamente la totalidad de las respuestas expresan una opinión negativa del cuestionario, lo cual revela la escasa valoración que los alumnos le atribuyen a este instrumento. Ellos criticaron el formato basado en una estructura de preguntas cerradas, lo que les impide expresar con amplitud sus valoraciones acerca del desempeño de los docentes. Las citas siguientes ilustran algunas percepciones de los alumnos: "El hecho de que contenga respuestas cerradas hace que piense que lo que opino no cuenta"; "Es demasiado estructurado, las preguntas son limitadas, estandariza a todos los docentes"; "Es un formato muy cuadrado y aunque existe un espacio para comentarios, es problemática la formulación de las preguntas". Como puede apreciarse en estas respuestas, algunos alumnos consideraron que el formato del cuestionario les induce a pensar que sus respuestas tienen escaso valor, o que conlleva a la estandarización de la enseñanza y deja fuera información valiosa que se podría obtener por otras vías.

El diseño cerrado del cuestionario propicia que los alumnos piensen que no cumple con el propósito para el cual ha sido creado y es suministrado. “...son muchas preguntas y como es de rellenar círculos, muchos los llenan sólo por llenar, sin poner una verdadera opinión”. Además de lo restrictivo de las preguntas cerradas algunos alumnos cuestionaron la calidad y pertinencia de las mismas. "Algunas preguntas son innecesarias o no aplican para todos los profesores... no están muy bien planteadas, a veces pienso que son inducidas. Es cansado y aburrido contestar preguntas que sólo se limitan al "excelente", "bueno", "regular", "siempre", "casi siempre”, "nunca”, etcétera.

Hubo voces críticas de alumnos que reconocieron contradicciones entre lo que se les enseña en la universidad y la existencia de ciertas prácticas educativas, lo cual generó una 
valoración negativa que va más allá del cuestionario, alcanzando incluso a la propia institución en su conjunto. "Es curioso que en nuestra formación nos digan que este tipo de encuestas son poco útiles y aun así las sigan implementando. Tú como alumno dejas de creer en tu universidad, rellenas las encuestas sin mirar, lo haces sólo por obligación".

Otra dimensión interesante que se puede rescatar de las opiniones vertidas se refiere a los resultados obtenidos con la aplicación del cuestionario. La mayor parte de los alumnos confía poco en que los resultados tengan algún valor para cambiar la enseñanza en el aula. "Si uno evalúa a un profesor negativamente, él continuará impartiendo su clase igual"; “...no sirve de mucho ya que los profesores siguen cometiendo los mismos errores..."; "Porque no se informa sobre los resultados y desconocemos los parámetros del desempeño de los profesores. No creo que funcione".

Aunque los alumnos quizá no tengan un conocimiento suficientemente desarrollado acerca del proceso de enseñanza, si parecen capaces de identificar que el cuestionario no incluye contenidos valiosos que, según su juicio, forman parte de una "buena enseñanza”, al tiempo que incorpora otros que resultan poco valiosos. "Se indaga poco sobre la comunicación interpersonal, que es muy importante para el aprendizaje, tampoco se toma en cuenta el tipo de proceso que cada profesor desarrolla... por ejemplo, se evalúa la asistencia, pero realmente lo que importa es la calidad de lo que el profesor enseña”. Estas declaraciones se relacionan con ciertos componentes de lo que en la bibliografía se identifica como las características de los sistemas de evaluación docente efectivos: a) situar al docente en el centro del proceso; b) incorporar múltiples fuentes de datos; c) reflejar múltiples observaciones e interacciones con el docente; c) incorporar la discusión de los datos de rendimiento del alumno; d) promover la reflexión y el análisis del docente; e) valorar el pensamiento del profesor sobre su docencia y su crecimiento profesional y; f) adaptar el proceso para que se ajuste al profesor individual y sus necesidades mientras cumple con todas las obligaciones legales y contractuales (Marx, 2011).

Sin embargo, existen matices, según sea la figura del docente a la que los alumnos tienen que evaluar y la etapa de la carrera en que se encuentran estudiando, aunque en general admitieron que el cuestionario es "tedioso y aburrido", que muchas veces lo contestan de forma mecánica y rutinaria sin detenerse a analizar las respuestas, esto no siempre ocurre así. "En ocasiones lo contesto por inercia, pero cuando se trata de maestros que sí me interesan (no me gustan/me gustan mucho como enseñan) lo hago detenidamente. Hay maestros que no son "ni muy muy, ni tan tan", entonces lo hago al tanteo"; "En los primeros trimestres todos se lo tomaban muy en serio, pero a estas alturas de la carrera la mayoría de los alumnos ya no pone atención a las preguntas y sólo lo rellenan de forma automatizada. La única excepción es cuando el grupo está inconforme con el docente”.

Aunque escasas, también se recuperaron algunas apreciaciones de los alumnos que podrían etiquetarse como "favorables" (a medias) acerca de este ejercicio periódico de aplicación del cuestionario.

$$
\begin{aligned}
& \text { Me gusta la idea de poder evaluar, es un ejercicio muy sano, sin embargo, no sé } \\
& \text { realmente para que se ocupa la información. En cuanto al cuestionario, creo que es } \\
& \text { muy completo, se puede obtener información muy valiosa y concisa. }
\end{aligned}
$$

Además, la mayor parte de las respuestas indica que los profesores no hablan con sus alumnos acerca del cuestionario de evaluación de su desempeño. Al interrogar a los alumnos acerca del por qué piensan que los docentes no comentan con ellos sobre este tema, las apreciaciones apuntaron a la escasa importancia que tiene para los propios 
docentes, el hecho de que no tiene consecuencias para ellos, o para evitar que sus opiniones influyan en las respuestas de los alumnos. Veamos algunas expresiones al respecto: "Porque no les afecta ni beneficia dicha encuesta, no les importa o ignoran su valor"; "Creo que no tiene ninguna repercusión, al menos ninguna importante"; "Supongo que para que podamos calificarlos libremente sin ningún tipo de presión”.

Incluso cuando algunos docentes han hablado con los alumnos acerca del cuestionario su valoración es negativa, lo que sin duda refuerza la apreciación que los educandos tienen en ese mismo sentido. "Opinan que la estructura es limitada en términos de una verdadera evaluación. No existen preguntas que dejen claro lo que aporta tal o cual profesor”; “...que el tiempo entre la aplicación y la entrega de los resultados es bastante grande. Nunca se habla de la importancia o el sentido que tiene para ellos".

Algunos alumnos atribuyeron la escasa importancia otorgada al cuestionario por parte del profesorado, al hecho de que ellos tienen asegurado su puesto de trabajo independientemente de los resultados de la evaluación. “...no le ven interés porque quizá tengan relaciones muy fuertes que les vale lo que digan los alumnos ya que eso no les quitará el puesto”.

En cuanto a las opiniones que los alumnos comparten con sus compañeros sobre del cuestionario, se aprecia que, al contrario de lo que ocurre con el profesorado, los alumnos si dedican tiempo a comentar entre ellos acerca del cuestionario y nuevamente se advierte que predomina una visión negativa.

No entendemos para qué lo contestamos. Nos disgusta contestarlo.

La mayoría de las veces se hace referencia a lo tedioso del proceso de aplicación y a lo repetitivo que llega a ser. También se habla de lo poco efectivo que resulta, pues no permite tener un panorama amplio de los procesos de aprendizaje en el aula.

Según se desprende de sus respuestas, lo que realmente disgusta a los alumnos es no conocer el sentido que tiene la aplicación del cuestionario.

No nos gusta responderlo porque no sabemos el papel que juega en la universidad.

Pienso que es para saber cómo está la planta docente, pero realmente no sabemos para qué es, cómo lo usan, si trae algún beneficio, en qué perjudica, etcétera.

Otros alumnos también comparten la idea de que el cuestionario de evaluación es poco útil y esgrimieron sus argumentos al respecto. "Es inútil, pues no conocemos los resultados y tampoco si se sanciona a los profesores que no tienen interés en su clase"; "Hemos hablado durante los 4 años de licenciatura sobre la ineficiencia de la evaluación ya que, a pesar de lo expresado por nosotros, hay profesores que no cambian su desempeño". Otros alumnos manifestaron su cansancio de tener que responder el cuestionario cada trimestre sin obtener a cambio ningún beneficio. "Es una pérdida de tiempo, no sirve para nada, es solo un trámite burocrático".

En ocasiones las opiniones se dividieron, mientras que algunos alumnos consideraron que el cuestionario es útil, la mayoría insistió en que carece de sentido. "Algunos coincidimos en que no sirve y otros que sí. La realidad es que sabemos poco acerca del uso que se le da"; "Es fastidioso, no permite una retroalimentación integral"; "Varios se quejan de que no quieren hacerlo, que lo contestan incluso sin leer las preguntas. Califican mal a un profesor, pero saben que eso no hará que el profesor cambie".

\subsection{Usos de los resultados de la evaluación docente}


La mayoría de las respuestas de los alumnos revelaron que desconocen los usos que la universidad hace de los resultados de la evaluación docente en la que ellos participan cada trimestre. No obstante, al preguntar acerca de los usos que ellos atribuyen a tales resultados, encontramos algunas respuestas que consideran sirve para conocer el nivel de competencia docente con que cuenta la universidad. "Supongo que sirve para asignar una calificación al desempeño de cada profesor y de esta forma determinar el nivel de competencia docente que tiene la universidad". Otras opiniones tienen que ver con la permanencia (o no) en la universidad de ciertos profesores, "No, no tengo conocimiento sobre su uso. Que a los maestros de planta no les perjudica en nada, pero para los que están por contrato o acaban de entrar sí influye lo que digan de ellos".

También se documentaron apreciaciones de los alumnos en el sentido de que la información obtenida es utilizada para cumplir con una función administrativa: la generación de estadísticas.

Sacar estadísticas, medir desempeño...

Sé que los cuestionarios se los hacen llegar a los profesores; ¿̨que es lo que pasa después?,

lo ignoro. Pienso que es sólo estadística; a menos que hubiera un resultado muy alarmante, en ese caso se haría una investigación o prevención hacia el profesor.

Otro conjunto de respuestas relacionadas con el uso que se hace de los resultados del cuestionario, revela que los alumnos son severos críticos sobre este punto, veamos algunas de sus valoraciones: “QQué hueva! ¡Otra vez! No veo los resultados, que hacen realmente con eso"; "Lo desconozco, solo sé que nos lo aplican en la $7^{\mathrm{a}}$ semana del trimestre. Es una evaluación de requisito, pero sin un fin. No tiene un objetivo, solo cumplir papeleo".

A pesar de la crítica negativa dominante, también se recuperaron algunas valoraciones positivas acerca de los usos de la información, las cuales apuntan a mejorar la calidad del profesorado mediante la puesta en marcha de acciones de formación docente. Aunque las respuestas dejan entrever un "cierto aire" de duda o recelo acerca de la efectividad de tales usos. "Supongo que es para que los alumnos mostremos el desempeño de cada profesor y de ese modo se pueda mejorar la calidad de la planta académica"; "Se supone que les dan cursos de docencia a los profesores que no obtienen buenos resultados en dicha evaluación"; "Según es para mejorar la docencia en la universidad. Solo como protocolo". Una función que se identifica claramente es el uso de la información para retroalimentar la tarea docente. "Me imagino que la utilizan para medir el desempeño del docente y así poder ayudarlo con el manejo del grupo"; "Creo que es para conocer si el profesor realiza bien su trabajo y darle cuenta de las fallas para poder mejorar”.

Por último, también hubo alguien que sugirió cómo superar el clima de indiferencia que reina entre los alumnos con respecto al cuestionario. "Hay una actitud de apatía, pero considero que si se informa a los alumnos sobre los procesos del cuestionario y si en realidad influye con los profesores se tendría otra actitud".

\subsection{Propuestas para evaluar el desempeño docente}

Un punto a destacar es que los alumnos no sólo reprocharon las inconsistencias del cuestionario y el uso que se hace de la información que genera, sino que también formularon propuestas para mejorar la evaluación del desempeño docente, ya sea modificando el cuestionario existente o bien reemplazándolo por otras técnicas e instrumentos más adecuados y pertinentes. Las respuestas de los alumnos se dividen básicamente en dos grupos: a) opiniones a favor de seguir empleando el cuestionario, 
aunque introduciendo cambios; y b) opiniones que proponen otras estrategias de evaluación más abiertas y cualitativas.

Respecto al primer grupo se recogieron algunas valoraciones,

Hay que cambiar el formato del cuestionario o reducirlo... es una buena opción, pero debería ser abierto, asi el profesor recibiría mayor información sobre sus aciertos y limitaciones.

Cuestionario con preguntas abiertas por internet, pero que se explique tanto su fin como su uso... es una herramienta, quizá no tan personalizado, pero que sirve muy bien para este fin. Propondría crear un buzón de quejas y sugerencias para conocer las opiniones de los estudiantes.

En cuanto a las voces que propusieron otras estrategias de evaluación más abiertas y cualitativas, emergieron las siguientes expresiones: "Entrevistas a alumnos voluntarios. Observaciones a alumnos y maestros en el aula”; "Una plática con el jefe de departamento sobre cada uno de los profesores"; "Interacción directa con el docente, pero con él dispuesto a hacerlo”. Incluso hubo quien pugnó porque las observaciones en el aula se hicieran sin previo aviso al docente, "Visitas de observación sorpresa a los profesores, para que se puedan observar los puntos a evaluar y se logren los cambios”. También hubo quien sugirió que el proceso de evaluación de la actuación del docente se centre en el modo en que enseña y evalúa a los alumnos: "la forma en que da su clase, que se tenga un control de la estructura del contenido que imparte, del tipo de evaluaciones que realiza, etc.”. Esto es importante porque la investigación ha demostrado que los docentes efectivos tienen un impacto mayor sobre la calidad de las experiencias educativas de los alumnos. Es primordial ofrecer a los profesores todo el apoyo que necesitan para hacer bien su trabajo porque cuando ellos tienen éxito los alumnos también tienen éxito (White et al., 2012).

También se identificó un grupo de declaraciones que no se refieren al cambio en la metodología de evaluación docente, sino que aluden a otros aspectos que los alumnos consideran importantes de incluir en una nueva perspectiva de la evaluación docente. "Alguna otra prueba a los docentes. Sin tener necesariamente que incentivar a los que obtengan buenos resultados”; “...que se haga de forma obligatoria en el salón de clases para tener la participación de la mayoría”.

\section{Conclusiones}

Se destaca que el proceso de evaluación de la docencia a través del cuestionario está asociado de forma prioritaria con el otorgamiento de estímulos económicos a los profesores y muy secundariamente con la mejora de la calidad de la docencia. Es por ello que “...esta evaluación no ha logrado resolver la cuestión de la calidad de la docencia, ni mejorado las habilidades pedagógicas de los académicos y, por ende, tampoco la calidad de la educación” (Arbesú y Comas, 2016, p. 133). Este hecho contrasta con una visión muy extendida en los últimos tiempos que vincula la evaluación con el mejoramiento de la educación. La evaluación de la docencia por sí misma no mejora nada, todo lo contrario, puede reforzar prácticas rutinarias y vacías de significado, lo que a la postre generará un rechazo de los participantes hacia este mecanismo, como ha quedado de manifiesto mediante los resultados obtenidos en esta investigación.

Entre las aportaciones principales de la investigación realizada se puede destacar que se trata de una investigación educativa de corte cualitativo que incorpora y recoge los puntos de vista de uno de los actores centrales del proceso formativo: los alumnos. El hecho de 
"dar voz" a quienes generalmente son silenciados en el proceso investigativo permite acceder a sus experiencias, vivencias, creencias y pensamientos acerca del tema investigado, lo cual aporta información valiosa para analizar y comprender mejor un proceso por naturaleza complejo como la evaluación de la docencia. Otro aporte importante es que los alumnos no sólo criticaron las incongruencias del cuestionario y el uso que se hace de los resultados, sino que también plantearon propuestas para mejorar la evaluación docente, ya sea modificando el cuestionario existente o bien sustituyéndolo por otras técnicas e instrumentos más cualitativos. Además, este estudio revela el malestar de los estudiantes y contrasta estos resultados con otros planeamientos teóricos y estudios previos, lo que evidencia la necesidad de mejorar los sistemas de evaluación docente.

Se acepta que no es posible mejorar la enseñanza y su evaluación dando la espalda a los alumnos, pues es a ellos a quienes va dirigida la enseñanza y por tanto quienes ocupan una posición privilegiada para brindar información acerca de su experiencia en las clases (Moreno, 2016). En este sentido,

\section{el rol protagónico y prioritario del propio estudiante en el proceso de enseñanza y aprendizaje le confiere no solo el derecho, sino la legitimidad, de ser un actor presente y válido a la hora de opinar y de juzgar la calidad de la enseñanza que recibe y, en tal sentido, analizar la práctica, el estilo y las actitudes de sus profesores. (Román, 2010, p. 5)}

Como ha quedado de manifiesto, los alumnos han hablado bien y fuerte acerca de una práctica que califican de rutinaria y carente de sentido: el uso del cuestionario como herramienta para valorar el desempeño de sus profesores. No obstante, hay quien considera que otorgar demasiado peso a las opiniones de los alumnos en relación con el desempeño de sus docentes entraña ciertos riesgos, toda vez que se les concede excesivo poder para decidir sobre aspectos que no siempre están cualificados para evaluar, lo cual puede conducir a una disminución de la calidad de la enseñanza (García, 2008). Sin embargo, también existen otras voces que discrepan de esta visión al señalar que no se trata de pedir a los alumnos que juzguen el nivel de conocimientos o el dominio de los contenidos disciplinares que tienen sus docentes, pues carecen de los conocimientos y criterios suficientes para hacerlo. Pero los alumnos si pueden identificar qué características de sus maestros, qué estrategias y estilos de enseñanza inciden en su motivación para aprender y sus logros. Ellos brindan información valiosa acerca de los factores que los inducen a permanecer o abandonar la escuela, los que en muchos casos se relacionan con los profesores, con sus expectativas y actitudes, con su apoyo o su desinterés (Román, 2010).

Una de las limitaciones de este trabajo estriba en que al tratarse de un estudio de caso los resultados no se pueden generalizar a otros casos cuyo contexto y condiciones sean distintos al investigado. Entre los futuros estudios que se podrían realizar a la luz de los resultados está el explorar las perspectivas de todos los demás participantes en el proceso de evaluación docente, especialmente de las autoridades educativas universitarias, figuras que han sido poco investigadas no sólo en México sino en el ámbito internacional (Moreno y Arbesú, 2015).

Dado que el objetivo de este artículo consistió en analizar las perspectivas de los alumnos de licenciatura con respecto al cuestionario de opinión con el que evalúan el desempeño de sus profesores, se encontró que existen distintas valoraciones, pero, en general, se aprecia cierto consenso que juzga negativamente la pertinencia y utilidad de dicho instrumento como una estrategia cierta para valorar el quehacer de los docentes en el aula. 
De esto se infiere la necesidad de repensar el diseño y uso del cuestionario como una herramienta eficaz para dar cuenta de la calidad de la enseñanza universitaria.

A modo de colofón sólo resta mencionar que, si se espera acabar con el hartazgo de los alumnos en relación con el cuestionario de evaluación docente, se tendrán que explorar otras opciones metodológicas más auténticas e integrales de evaluación del desempeño docente, pues una vez reconocida la complejidad del proceso de enseñanza resulta paradójica simplificar su valoración mediante un cuestionario cerrado.

\section{Referencias}

Arbesú, M. I. y Comas, O. (2016). Representaciones sociales estudiantiles y evaluación docente. Reencuentro. Análisis de Problemas Universitarios, 71(27), 123-136.

Arbesú, M. I. y García, J. M. (2017). La evaluación docente en jaque: La visión de los funcionarios universitarios. Revista Iberoamericana de Evaluación Educativa, 1O(2), 171-185. https://doi.org/10.15366/riee2017.10.2.009

Comas, O. y Lastra, R. (2010). Institucionalización y programas de estímulo económico a profesores universitarios en México: Un análisis por intersticios. Revista de la Educación Superior, 39(155), 81-98.

Cordero, G. y González, C. (2016). Análisis del modelo de evaluación del desempeño docente en el marco de la reforma educativa mexicana. Archivos Analíticos de Políticas Educativas, 24(46), art 5. https://doi.org/10.14507/epaa.v24.2242

Cordero, G., Luna, E. y Patiño, N. (2013). La evaluación docente en educación básica en México: Panorama y agenda pendiente. Sinéctica, 41, 1-19.

Cuevas, Y. y Moreno, T. (2016). Políticas de evaluación docente de la OCDE: La experiencia de la educación básica en México. Archivos Analíticos de Políticas Educativas, 24(120), art 3. http://dx.doi.org/10.14507/epaa.24.2283

Darling-Hammond, L. (2013). Getting teacher evaluation right. What really matters for effectiveness and improvement. Nueva York, NY: Teacher College Press.

Darling-Hammond, L. y McLaughlin, M. (2003). El desarrollo profesional de los maestros. Nuevas estrategias y políticas de apoyo. Ciudad de México: SEP.

Escudero, J. M. (2002). La reforma de la reforma. ¿Qué calidad, para quiénes? Barcelona: Ariel.

Fullan, M. (1993). Changes forces. Probing the depths of educational reform. Londres: Falmer Press.

García, J. M. (2008). El proceso perverso de la evaluación de la docencia en las universidades: Un balance inicial y apuntes para mejorarlo. Reencuentro. Análisis de Problemas Universitarios, 53, 9-19.

Hargreaves, A. (1996). Profesorado, cultura y posmodernidad. Madrid: Morata.

Jackson, P. (2002). Práctica de la enseñanza. Buenos Aires: Amorrortu.

Lawn, M. y Ozga, J. (2004). La nueva formación del docente. Identidad, profesionalismo y trabajo en la enseñanza. Barcelona: Pomares.

Marx, G. E. (2011). Teacher evaluation. Recuperado de http://www.educationpartnerships.org/

Moreno, T. (2006). La colaboración y la colegialidad docente en la universidad: Del discurso a la realidad. Perfiles Educativos, 38(112), 98-130. 
Moreno, T. (2010). Lo bueno, lo malo y lo feo: Las muchas caras de la evaluación. Revista Iberoamericana de Educación Superior, 1(2), 84-97.

Moreno, T. (2016). Evaluación del aprendizaje y para el aprendizaje. Reinventar la evaluación en el aula. Ciudad de México: Universidad Autónoma Metropolitana-Cuajimalpa.

Moreno, T. y Arbesú, M. I. (2015). Visiones de las autoridades universitarias sobre la evaluación docente. En Institut de Ciències de l'Educació Josep Pallach (Org.), Memorias del V Congreso Internacional. UNIVEST' 15. Los retos de mejorar la evaluación (pp. 454-458). Girona: Universidad de Girona.

Murillo, F. J. (2003). El movimiento teórico-práctico de mejora de la escuela. algunas lecciones aprendidas para transformar los centros docentes. REICE. Revista Iberoamericana sobre Calidad, Eficacia y Cambio en Educación, 1(2), 5-23.

Murillo, F. J. (2007). Evaluación del desempeño y carrera profesional docente. Una panorámica de América y Europa. Santiago de Chile: UNESCO.

Nevo, D. (1997). Evaluación basada en el centro. Un diálogo para la mejora educativa. Bilbao: Mensajero.

Román, M. (2010). La voz ausente de estudiantes y padres en la evaluación del desempeño docente. Santiago de Chile: PREAL.

Rueda, M. y Díaz Barriga, F. (Coords.). (2011). La evaluación de la docencia en la universidad. Perspectivas desde la investigación y la intervención profesional. Ciudad de México: IISUEUNAM.

Tejedor, F. J. y Jornet, J. M. (2008). La evaluación del profesorado universitario en España. Revista Electrónica de Investigación Educativa, 10 (num. especial), 1-29.

White, B. R., Cowhy, J., Stevens, W. D. y Sporte, S. E. (2012). Implementing the next generation of teacher evaluation systems. Lessons learned from case studies in five Illinois districts. Chicago, IL: The University of Chicago.

\section{Breve CV del autor}

\section{Tiburcio Moreno Olivos}

Doctor en Pedagogía por la Universidad de Murcia. Profesor Investigador Titular del Departamento de Tecnologías de la Información de la Universidad Autónoma Metropolitana-Cuajimalpa de la Ciudad de México. Miembro del Sistema Nacional de Investigadores del CONACYT y del Consejo Mexicano de investigación educativa (COMIE). Ha dirigido 7 tesis de maestría y 12 de doctorado en Pedagogía y Ciencias de la Educación. Su trayectoria de investigación y publicaciones abarca varias líneas de trabajo: a) La evaluación educativa, y particularmente la evaluación del aprendizaje, b) La educación secundaria, c) Currículum y d) Procesos de formación docente. ORCID ID: o000-0002-0392-6621. Email: tmoreno@correo.cua.uam.mx 